Estudos de Psicologia

2001, Vol 18, no 3, 33-42

\title{
O Brincar no Hospital: Análise de Estratégias e Recursos Lúdicos Utilizados com Crianças
}

\author{
Rita Márcia Aragão ${ }^{1}$ \\ Maria Rita Zoega Soares Azevedo
}

\begin{abstract}
Esta pesquisa buscou caracterizar a preferência de recursos e atividades lúdicas de acordo com faixas etárias e, de modo geral, investigar a efetividade desses recursos e atividades no atendimento a crianças hospitalizadas, facilitando a expressão de sentimentos e aquisição de conceitos relacionados ao contexto hospitalar. A população-alvo consistiu de trinta e seis sujeitos, sendo vinte e três do sexo masculino e treze do sexo feminino, com idade entre nove meses a doze anos, internados no setor de enfermaria pediátrica de um hospital público da cidade de Londrina. A coleta de dados foi realizada através do relato das observações de atividades desenvolvidas e do material lúdico utilizado com cada um dos sujeitos. Verificou-se a relação entre a freqüência de utilização de um determinado material e a atividade para cada faixa etária o Concluiu-se que atividades ou recursos puderam ser explorados em determinadas idades, enquanto outras foram aceitas em diversas faixas etárias, contudo com objetivos diferentes. De modo geral, pode-se dizer que os recursos foram efetivos, possibilitando a expressão de sentimentos relacionados à hospitalização.
\end{abstract}

Palavras-chave: crianças hospitalizadas, hospitalização infantil, psicologia hospitalar, estratégias lúdicas.

\begin{abstract}
Playing at the Hospital: Analysis of Strategies and Resources of the Used with Children These research had the objectives to characterize the preference of resources and activities of the games in agreement with the age groups and, in general to investigate the effectiveness of those resources and activities in the attendance to hospitalized children, facilitating the expression of feelings and acquisition of concepts related to the hospital context. The target people were thirty six subjects, twenty three male and thirteen female, with ages between nine months and twelve years old, interned in the children ward section of a Londrina's Public Hospital. The data collection was accomplished through the observations reported among the activities developed, and the gaming material used with one of the subjects. Was verifyed the relation between the frequency of using a kind of material and activity for each age groups. Deduced that activities or resources they could be explored in certain ages, while others were accepted in several age groups, however, with different objectives. In general, it can be said that the resources were effective, making possible the expression of feelings related to the hospitalization.

Key words: hospitalized children, infantile hospitalization, hospital psychology, activities of the games.
\end{abstract}

1. Discente do Curso de Especialização em Psicoterapia na Análise do Comportamento da Universidade Estadual de Londrina-UEL

Endereço para correspondência: Rua Itálial, 254, Jardim Igapó, Londrina, PR - CEP: 86046-130.

2. Docente da UEL.

Endereço para correspondência: Rua Prefeito Hugo Cabral, 1062, aptô142, Londrina, PR - CEP: 86020-917.

Pesquisa derivada de monografia realizada no curso de Especialização em Psicoterapia na Análise do Comportamento (UEL), cuja coleta de dados foi realizada em hospital público de Londrina. 


\section{Introdução}

\section{A importância do brincar}

O brincar tem sido o foco de interesse de muitas pesquisas que consideram essa atividade necessária à aprendizagem de conceitos em crianças (Azevedo, 1999; Whaley \& Wong, 1989; Bontempo, 1986; Lindquist, 1992; Santos, 1997).

Whaley \& Wong (1989) consideram que crianças podem desenvolver conceitos relacionados ao seu ambiente e estratégias para lidar com ele através do brincar, praticando como lidar com as situações complicadas da vida, comunicando-se e estabelecendo relações satisfatórias com outras pessoas, estimulando e aprimorando seu desenvolvimento social e suas relações interpessoais.

Conforme os autores citados anteriormente, crianças pequenas participam de brincadeiras simples e imitativas; pré-escolares aprendem e divertem-se com jogos convencionais, iniciando com brincadeiras ritualistas e progredindo para jogos com regras, competitivos e cooperativos.

Segundo Guimarães (1988) brincar implica na interação com o ambiente, geralmente de modo relaxado e envolvendo afeto positivo. Crianças brincam com temas relacionados ao seu ambiente diário, e se esse contexto muda, as brincadeiras também podem mudar.

\section{A criança hospitalizada}

A condição de hospitalização desempenha um papel importante na manutenção da saúde de crianças enfermas. No entanto, nessa situação podem surgir condições que dificultam a adaptação a esse contexto (Laurent \& Rumeu, 1990).

Gaetan (1997) discute que tanto a hospitalização como a doença podem provocar estresse à criança. Angerami (1994) considera que a criança pode entrar num nível de sofrimento emocional e físico, em decorrência da hospitalização. Essa situação pode criar ameaças reais ou imaginárias, que podem ser expressas através do medo de médicos, choro, agressividade, dependência, ansiedade, depressão, distúrbio do sono, evitação dos procedimentos médicos e outras formas de respostas (Domingos, 1993).

A hospitalização modifica a vida da criança, pois ela sofre com a doença, é afastada da família, da escola e dos objetos pessoais, perdendo grande parte de suas referências. Além disso, pode-se encontrar no hospital um ambiente hostil, com procedimentos invasivos e uma atmosfera geralmente tensa e ameaçadora (Sagguese \& Maciel, 1997).

Quando se trata da hospitalização infantil, deve-se considerar o ambiente novo, as pessoas que cuidarão dela e a doença como condições estranhas ao paciente. Assim, a equipe do hospital precisa estar hábil para lidar com as crianças e seus pais, pois, caso contrário, a internação pode ficar complicada. A forma como a hospitalização e a doença são vivenciadas pode influenciar no surgimento de dificuldades relacionadas à adaptação (Gaetan, 1997).

Laurent \& Rumeu (1990) afirmam que fatores pessoais (idade, desenvolvimento biopsicossocial do paciente, natureza e grau de severidade da enfermidade, experiências prévias com médicos e hospitais), familiares (maneira dos pais, irmãos, familiares e companheiros responderem à hospitalização) e hospitalares (contato com ambiente estranho, mudanças do pessoal do hospital, duração da internação, características e organização do centro hospitalar, ausência de informações e privação cultural, social e escolar) podem interferir na hospitalização infantil.

Para Guimarães (1988) a falta de variedade, de estrutura, de organização dos estímulos e atividades para a interação da criança são fatores que dificultam a adaptação ao contexto hospitalar. 
Guimarães (1988) e Azevedo (1999) descreveram a hospitalização como uma oportunidade para a criança e a família enriquecerem seu repertório comportamental. Para isso, profissionais da área de saúde devem considerar a criança hospitalizada como um todo biopsicossocial em crescente desenvolvimento.

A preparação para a hospitalização deve ter como objetivos reduzir os efeitos negativos que a mesma exerce sobre a criança e fornecer informações que a deixem menos vulnerável às experiências negativas (Laurent \& Rumeu, 1990; Méndez, Ortigosa e Pedroche, 1996).

Para Ortigosa, Méndez \& Quiles (1996), a falta de informações relacionadas à situação hospitalar pode tomar-se potencial estressor. Porém, a informação precisa e adequada ajuda a diminuir o estresse.

\section{O brincar no contexto hospitalar}

O brincar é essencial para a criança, inclusive hospitalizada. Mesmo em situações estressantes ela pode beneficiar-se dele, possibilitando relaxamento e compreensão da situação (Ribeiro, 1998).

Azevedo (1999) e Méndez et al. (1996) falam da importância de tomar as enfermarias pediátricas mais adequadas ao serviço prestado às crianças, salientando que os pacientes devem ser preparados para a hospitalização e procedimentos médicos.

Pesquisas demonstram que a preparação para esses procedimentos amenizam o medo e a ansiedade, podendo exercer efeitos positivos sobre a recuperação do paciente. Atividades lúdicas devem ter a função de intervenção terapêutica no contexto hospitalar porque, brincando, a criança pode incrementar seu repertório comportamental e experimentar diferentes respostas de ajustamento ao meio (Whaley \& Wong, 1989; Domingos, 1993; Sagguese \& Maciel, 1997).
Lindquist (1993) fala do direito da criança à informação sobre sua enfermidade e dos procedimentos médicos a que será submetida. $\mathrm{O}$ brinquedo pode ser utilizado como recurso para informá-la e prepará-la para esses procedimentos.

Domingos (1993) discute que é preciso esclarecer o processo aos pais e criança (explicando o que vai acontecer), encorajar expressões emocionais e estabelecer clima de confiança na relação com a equipe hospitalar.

Devem ser levados em conta idade, nível de desenvolvimento e o local onde possa brincar, ajustando o programa de preparoàs necessidades da mesma e à sua capacidade de entendimento (Domingos, 1993; Lindiquist, 1975).

O brincar pode ser explorado para proporcionar oportunidade de desenvolvimento humano, pois é uma forma de interagir com o ambiente; a criança interage com seu meio à medida em que oportunidades Ihe são oferecidas, provocando modificações no seu repertório comportamental e na natureza funcional do meio.

"O atendimento por profissionais qualificados na ciência do comportamento pode prover, junto à equipe pediátrica, recursos técnicos para minimizar possíveis perdas ou atrasos no desenvolvimento que poderia sofrer uma criança em situação hospitalar", considerando sua condição socioeconômica e religiosa, ambiente físico e características das fases do desenvolvimento infantil (Guimarães, 1988).

Para organizar atividades, deve-se considerar a idade da criança, porque, quanto mais nova, maior a dificuldade em compreender a situação e exprimir sentimentos (Azevedo, 1999). Crianças em idade escolar devem ter melhor repertório para verbalizar medo e dor. $\mathrm{O}$ fornecimento de informações e oportunidades lúdicas diversas parecem ser eficientes (Domingos, 1993). Assim, a presença de brinquedos adequados às faixas etárias pode melhorar a condição de preparo para o contexto hospitalar. 
O mesmo material pode ser usado com crianças de idades diferentese de diferentesmaneiras. Tais materiais devem ser usados em atividades que desafiam, sem produzir frustração elevada.

Lindquist (1992) sugere que criança e pais devem encontrar no hospital um espaço dedicado ao jogo. Dessa forma, criança e pais podem perceber que há preocupação com a saúde global do paciente.

A Psicologia Hospitalar é considerada um campo recente de intervenção. Há necessidade de verificar se os recursos e atividades utilizadas com as crianças hospitalizadas são efetivos, diminuindo conseqüências negativas advindas dessa condição.

Este trabalho buscou caracterizar a preferência de recursos e atividades lúdicas, de acordo com as faixas etárias, identificar e analisar a efetividade destes, facilitando expressão de sentimentos e aquisição de conceitos relacionados ao contexto hospitalar.

Tal condição pode fazer com que a Psicologia contribua na melhora do repertório, socializando a criança no contexto hospitalar, generalizando tais ganhos para as diversas situações.

\section{Metodologia}

\section{Local}

O trabalho foi desenvolvido em um hospital público da cidade de Londrina, PR.

\section{População-alvo}

Trinta e seis pacientes com idade entre 09 meses e 12 anos, internados no setor de enfermaria pediátrica no referido hospital, com nível socioeconômico baixo, foram submetidos ao trabalho proposto.

Onze dos sujeitos pertenciam à faixa etária entre os 2 anos, dez à faixa etária entre 3 e 5 anos, quatorze crianças entre 6 e 8 anos, e uma criança na faixa etária entre 9 e 12 anos.

\section{Recursos humanos}

Participaram da pesquisa uma aluna do curso de pós-graduação em Psicoterapia na Análise do Comportamento, três estagiárias de Psicologia da UEL, sendo supervisionadas por uma docente do departamento de Psicologia.

\section{Recursos materiais}

Os brinquedos foram: boneca (Babytoys), massa de modelar (Acrilex), carrinho (Toys), chocalhos (BabyToys; Toys Super), bichinhos emborrachados (Walt Disney), bola de espuma;jogos: dominó (Omotcha Indústria de Brinquedos), boliche (Móbile), quebra-gelo (Grow), quebra-cabeças (Grow), basquete (Aim N' Shoot Basketball Set); material sucata; livro de história (O coelhinho azul e o remédio mágico - ed. EKO); materiais de desenho: giz de cera (Acrilex), sulfite (Chamequinho), lápis de cor (Faber-Castel), tinta guache; dobradura e materiais hospitalares (luvas cirúrgicas, gaze, esparadrapo e bolsa de primeiros socorros).

O livro infantil foi selecionado porque referia-se à situação específica de enfermidade.

\section{Procedimento}

A pesquisa foi realizada no período de junho a outubro de 1999. Os sujeitos foram selecionados através de fichas de identificação utilizadas no próprio hospital, levando-se em consideração a idade de cada um. Estagiárias esclareceram os objetivos do trabalho e solicitaram permissão aos responsáveis para que a criança pudesse participar da pesquisa. Os pais foram informados de que estavam livres para permanecerem ou não junto à criança. $A$ intervenção foi desenvolvida por estagiárias do curso de Psicologia da UEL, durante um dia no período de internamento de cada criança.

O trabalho foi realizado na enfermaria do hospital, oferecendo-se às crianças diversos brinquedos para que optassem pelo material de 
sua preferência. Os recursos foram pré-selecionados segundo os critérios utilizados por Azevedo (1999) e os brinquedos foram oferecidos de acordo com a faixa etária de cada criança: 0-2 anos (bichinhos emborrachados, chocalho, carrinho, quebra-gelo, boneca, bola de espuma); 3-5 anos (materiais de desenho, quebra-cabeças, carrinho, massa de modelar, boneca, livro infantil, jogo de basquete, materiais hospitalares); 6-8 anos (materiais de desenho, carrinho, dominó, quebra-cabeças, sucata, boliche, massa de modelar, dobradura, materiais hospitalares); 9-12 anos (materiais de desenho, dominó, quebra-cabeças, dobradura, livro infantil, materiais hospitalares).

Os dados foram descritos pelas estagiárias através de registro cursivo após cada intervenção e analisados posteriormente.

\section{Análise de dados}

Os dados coletados foram analisados quantitativamente (através de porcentagens) e qualitativamente (descrição das atividades realizadas).

Para facilitar a análise, as crianças foram divididas em subgrupos, de acordo com cada faixa etária, segundo sugestão de Azevedo (1999).

\section{Resultados}

Como o objetivo do trabalho foi considerar e descrever os materiais utilizados para cada faixa etária, não se explicitou o que foi usado por cada criança especificamente. As atividades foram oferecidas por estagiárias, que observaram 0 comportamento da criança em relação ao objeto oferecido.

Na faixa etária compreendida entre 0-2 anos pôde-se constatar que o carrinho, o chocalho e os bichinhos emborrachados foram os que obtiveram maior freqüência de utilização nas atividades propostas, com 36\%, 45\% e 54\% respectivamente.
Nessa faixa etária, as intervenções ocorreram com $100 \%$ de freqüência na presença das mães, que receberam informações e orientações em relação à situação hospitalar e ao desenvolvimento da criança. Na maioria das vezes, as mesmas atividades e materiais foram utilizados para ambos os sexos.

Com bichinhos emborrachados e chocaIhos procurou-se produzir estimulação tátil, visual e auditiva, através da demonstração do brinquedo à criança, buscando chamar sua atenção, fazendo movimentos e sons. Observou-se que, no início, a criança apenas olhava, mas depois passou a interagir, mantendo contato visual, sorrindo, manuseando o brinquedo e produzindo sons.

Tal recurso foi também utilizado para contar histórias, incluindo noções sobre procedimentos médicos realizados e sua função. Observou-se que, apóstal atividade, crianças que se submetiam à inalação segurando o brinquedo, diminuíam respostas relacionadas ao choro e de oposição.

Bonecas, carrinhos, quebra-gelo e bola de espuma também foram utilizados no preparo para procedimentosmédicosecompreensãodeaspectos relacionados à hospitalização. Tal material também foi explorado através do brincar livre.

Passar o carrinho pelo braço da criança, simulando uma "estrada" foi uma das estratégias utilizadas, e tal condição produzia respostas relacionadas ao sorriso, contato visual e emissão de sons. Tais comportamentos também foram observados durante o martelar blocos do quebra-gelo e o jogar bola.

$\mathrm{Na}$ faixa etária compreendida entre 3-5 anos, materiais de desenho (papel, giz de cera, lápis de cor) e quebra-cabeças foram utilizados em $60 \%$ das intervenções; o carrinho em 50\% delas, a massa de modelareabonecaem $30 \%$ das atividades. Nessa faixa etária, 80\% dos acompanhantes deixaram as crianças com as estagiárias, saindo do quarto durante a intervenção. 
Foi incentivado o desenho livre nesta situação. Constatou-se que produziam desde rabiscos no papelaté desenhos mais elaborados. Ao término do desenho, foram questionadas sobre o que haviam desenhado e seu significado. Algumas crianças tiveram dificuldade para verbalizar o que tinham feito. Outras relataram fatos sobre suas vidas e sentimentos em relação à hospitalização. Os desenhos foram colocados na parede da enfermaria e cedidos para as crianças após a alta. O livro de história foi lido e questionado a partir do tema, visando a compreensão do conteúdo. A atividade foi finalizada com a solicitação de desenhos referentes à história lida.

Quebra-cabeças foram montados com o auxílio da estagiária e as crianças tiveram oportunidade de conversar sobre suas vidas e relatar sentimentos frente à hospitalização. Com as bonecas, crianças verbalizaram sobre suas vidas dentro e fora de hospital. Tal material também foi utilizado para demonstrar procedimentos médicos como: colocar curativos, puncionar veias, tomar medicamentos e aplicar injeções.

Com carrinhos, as crianças construíram circuitos de corrida, simulando acidentes reais que tinham acontecido com elas. Enquanto brincavam, também verbalizaram sobre suas vidas $\mathrm{e}$ sentimentos percebidos naquele contexto. Houve grande preferência pela utilização de carrinhos nessa faixa etária, provavelmente pelo fato de a maioria dos sujeitos ser do sexo masculino e culturalmente tal brinquedo ser bastante incentivado.

Com a massa de modelar, através de manipulação livre, buscou-se incentivar a interação entre estagiária e paciente, pois podiam construir juntos, utilizando o mesmo material. As crianças foram questionadas acerca das cores e formas utilizadas.

Materiais hospitalares foram usados buscando aumentar o contato dos pacientes com luvas cirúrgicas, bolsa de primeiros socorros, gaze e esparadrapo para que pudessem manuseá-los de forma menos aversiva, incentivando expressão de sentimentos e uso de fantasias em relação à hospitalização. As crianças questionaram sobre aspectos relacionados ao contexto hospitalar e suas próprias condições de saúde. Tais questões foram respondidas e dramatizadas através do uso deste material.

Entre os sujeitos de 6-8 anos, materiais de desenho foram os mais utilizados ( $64 \%$ das intervenções). Nessa faixa etária, $85 \%$ dos acompanhantes também saíram do quarto durante a intervenção.

Materiais de desenho foram utilizados incentivando-se o desenho livre envolvendo situações relacionadas ao dia-a-dia da criança. Desenhos também foram feitos, com a iniciativa da própria criança, para presentear outras crianças e membros da família. Em muitas situações, as crianças escreveram dedicatórias expressando o que sentiam pela pessoa a quem era dedicado o desenho. Desenhos também foram colocados na parede ou levados para casa após a alta.

Enquanto executavam essa atividade, as crianças puderam relatar sobre suas vidas e expressar sentimentos através da escolha de cores, relacionando-as com o que sentiam naquele momento.

Com carrinhos, as crianças simulavam circuitos de corridas e trombadas, demonstrando acidentes acontecidos na família. Também se explorou o verbalizar sentimentos acerca de procedimentos médicos utilizados, bem como a função de cada um deles.

O dominó foi usado explicitando as regras do jogo, incentivando tanto competição como cooperação. Pôde-se constatar o padrão comportamental das crianças frente a tal atividade (trapacear, desistir, ajudar). Enquanto jogavam falavam sobre suas vidas e expressavam sentimentos diante da hospitalização. $O$ boliche também foi utilizado buscando tais objetivos. 
Quebra-cabeças foram montados com o auxílio de estagiárias, buscando favorecer a interação. Tal condição pode ser efetiva no sentido de evitar que o paciente se frustre por não conseguir montar, o que não seria indicado para a condição de hospitalização. Tal atividade oportunizou verbalizações sobre suas vidas, bem como sentimentos envolvidos no contexto.

O material sucata foi utilizado para que a criança também tivesse oportunidade de criar brinquedos, generalizando tal padrão comportamental para outras situações. Após terem construído os próprios brinquedos, demonstraram satisfação, exibindo o que tinham feito para outras pessoas.

A massa de modelar foi utilizada explorando-se a criatividade e dramatização de situações. Tal recurso demonstrou ser efetivo para expressão de sentimentos nesse contexto.

O sulfite foi explorado para confecção de animais ou objetos através de dobraduras. As crianças participaram observando as estagiárias e auxiliando-as nas dobraduras. A seguir, foram incentivadas a pintar e colar a produção na parede da enfermaria.

Entre 9-12 anos, apenas o dominó foi utilizado no desenvolvimento da atividade. Nessa faixa etária, as crianças não ficavam na presença de acompanhantes. Observou-se, nessa situação, aumento na freqüência de verbalizações sobre aspectos de sua vida e seus sentimentos em relação à condição hospitalar.

\section{Discussão}

A exploração de recursos lúdicos no contexto hospitalar foi tema de interesse dessa pesquisa porque tem demonstrado efetividade no trabalho com crianças, facilitando compreensão dos sentimentos dos pacientes pediátricos, bem como fornecendo informações adaptadas à idade de cada um.
Para Azevedo (1999), quanto mais nova, maior a dificuldade da criança em compreender a situação e expressar o que sente. A utilização de tais recursos pode facilitar para que o profissional trabalhe com ela, principalmente dentro do hospital.

Através da análise dos dados, observou-se que a faixa etária entre 0-2 anos utilizou com maior freqüência bichinhos emborrachados e chocalhos. Tal condição pode estar relacionada ao fato de o material permitir oportunidade para trabalhar estimulação tátil, visual e auditiva. Crianças pequenas participaram de brincadeiras simples e imitativas, necessitando receber tal estimulação, pois estariam iniciando a exploração e a descoberta do mundo, mesmo hospitalizadas (Azevedo, 1999).

Com sujeitos de aproximadamente 2 anos, explicaram-se procedimentos médicos, a função de cada um, utilizando jogos direcionados a outras faixas etárias. Segundo Lindquist (1975), o jogo deve ser adaptado ao nível de desenvolvimento da criança e pode ser utilizado sem seguir suas instruções, adaptando a atividade à condição de cada uma. Assim, constatou-se que tal recurso auxiliou na interação entre crianças e profissionais, possibilitando a expressão de sentimentos através de sorrisos e verbalizações.

O quebra-gelo, carrinho e bola de espuma foram utilizados também com o objetivo de promover relaxamento e a expressão de sentimentos. Guimarães (1988) e Ribeiro (1998) afirmaram que o comportamento de brincar implica na interação com o ambiente, de modo relaxado e envolvendo afeto positivo, possibilitando maior compreensão da situação estressante. Consideramos que tais formas de brincar favoreceram para que a criança vivenciasse seus medos e ansiedades, proporcionando-lhe desenvolvimento no repertório para enfrentar condições adversas. 
Outro aspecto considerado importante foi a orientação às mães e a importância de seu incentivo e estimulação, para que a criança não tenha seu desenvolvimento comprometido. Lindquist (1992) sugeriu que, com o espaço dedicado ao brincar no hospital, não só a criança, mas os pais podem perceberque háuma preocupação com a saúde global da criança.

Em crianças com idade entre 3-5 anos, verificou-se maior freqüência na utilização de materiais de desenho e quebra-cabeças. Nessa fase, a criança estaria aprimorando a coordenação motora fina (Gesell, 1985) e, talvez devido a esse fator, tal atividade tenha sido selecionada por muitas crianças nessa faixa etária. Tal fato também tem relação com a condição de que as crianças já freqüentavam a pré-escola, e possuíam repertório desenvolvido para desenvolver tais atividades. Esses materiais devem ser usados em atividades que desafiam, sem produzir frustração elevada.

Entre 6-8 anos, as crianças também optaram por materiais relacionados ao desenho. Considerou-se que as crianças nessa faixa etária estavam freqüentando a escola e tinham conhecimento das possibilidades desse material (Piaget, 1964). A criatividade da criança foi incentivada para que pudessem elaborar atividade onde a expressão de sentimentos fosse mais facilmente trabalhada.

Nessa fase também estavam presentes a utilização de jogos com regras e brincadeiras ritualistas e perpetuantes, como Whaley \& Wong (1989) já definiram. Tais recursos foram explorados como estratégias para o trabalho favorecendo a interação, possibilitando a verbalização sobre aspectos relacionados à sua vida e sentimentos.

$\mathrm{Na}$ faixa entre 9-12 anos, observou-se que a criança utilizou predominantemente o dominó e demonstrou compreender regras e instruções relativas ao jogo. Segundo Whaley \& Wong (1989), nesta fase as crianças progridem para jogos com regras, competitivos e cooperativos.

Constatou-se que é necessário que se explorem vários materiais para que a criança demonstre interesse por algum deles, exigindo do estagiário elevado investimento pessoal para conseguir desenvolver qualquer atividade. Dessa forma, o profissional deve estar atento para orientar atividades que se adaptem e auxiliem a criança a lidar com dificuldades. Quando as crianças não demonstram interesse pela brincadeira e não procuram interagir, deve-se levar em conta a possibilidade de dificuldades relacionadas a fatores familiares, pessoais e hospitalares, já descritos por Laurent \& Rumeu (1990). Guimarães(1988) ressalta que a organização e estrutura do ambiente e atividades adequadas ao nível de desenvolvimento são necessárias para proporcionarem ao indivíduo condições de participação nas brincadeiras.

Lindquist (1993) descreveu a importância de cada paciente receber informações sobre sua enfermidade e procedimentos médicos, para diminuir a freqüência de respostas de medo e ansiedade, podendo colaborar com tais procedimentos. O fornecimento de orientações em relação a procedimentos médicos foi realizado através de linguagem adaptada ao nível de desenvolvimento do paciente. A criança tem direito a receber informações sobre sua enfermidade e os procedimentos médicos a que será submetida.

De modo geral, observou-se que todos os recursos contribuíram paraa expressão de sentimentos e vivência de situações relacionadas à hospitalização, verificadas através das verbalizações das crianças, em praticamente todas as faixas etárias. Observou-se que o lúdico contribuiu para a realização de procedimentos 
médicos. Diante de histórias onde o personagem teria de passar por tal condição, a criança parou de chorar e aceitou submeter-se a procedimentos clínicos. Lindquist (1992) ressaltou que brinquedos podem ser usados para esclarecer aspectos do contexto hospitalar, facilitando contato com a criança e aumentando sua colaboração.

Domingos (1993) demonstrou a importância de os pais participarem do processo junto à criança, pois podem desenvolver repertório alternativo para lidar com ela. Apesar disso, na faixa etária entre 3-12 anos, 84\% dos acompanhantes optaram por deixar os filhos sozinhos com as estagiárias durante as intervenções. Azevedo (1999) descreveu que, nessa situação, pode ser importante que pais também descansem e se envolvam com suas próprias coisas, facilitando, no retomo ao hospital, o convívio com a criança.

Percebeu-se que atividades como desenhar, jogar, modelar, conversar e recursos como carrinho, massinha e quebra-cabeças foram utilizados em várias fases de desenvolvimento da criança, mas de maneiras diferentes. Considera-se que as mesmas atividades podem ser utilizadas em diferentes fases do desenvolvimento da criança, só que com objetivos diferentes, adaptando-se ao sexo, nível de desenvolvimento e ambiente no qual o indivíduo está inserido.

Para a maioria das crianças, as atividades foram bem aceitas e favoreceram a interação, expressão de sentimentos e verbalizações. Constatou-se que carrinho, boneca e bichinhos emborrachados favoreceram a verbalização de histórias com temas relacionados a acidentes, hospitalização e morte, além de oportunidade de explicitarem procedimentos médicos.

De modo geral, percebeu-se que a maioria dos recursos foram efetivos, permitindo maior interação entre criança e profissional, verbalização de sentimentos e maior aceitação dos procedimentos médicos, além da diminuição na freqüência de respostas de choro diante da realização desses procedimentos.

Faz-se importante a realização de pesquisas que demonstrem a efetividade desses recursos na aceitação de procedimentos médicos e expressão de sentimentos relativos à hospitalização. De acordo com a análise do comportamento, expressar sentimentos, ou verbalizá-los, pode contribuir para a diminuição da ansiedade frente aos procedimentos médicos, porque pode diminuir o sentimento de incontrolabilidade diante da situação de hospitalização, além de modificar a função da condição estimuladora. Tal condição deveria ter como objetivo a manutenção da saúde da criança e o desenvolvimento de repertório útil, quando generalizado para lidar com condições de vida adversas, além de aumentar a socialização no contexto hospitalar.

\section{Referências Bibliográficas}

Angerami, V. A. (1994). O psicólogo no hospital. In: V. A. Angerami, Psicologia hospitalar. São Paulo: [s.n.]

Azevedo, M. R. Z. S. (1999). O papel e importância do lúdico para profissionais da saúde: Análise de jogos e brincadeiras no contexto hospitalar. (Dissertação de Mestrado em Educação, Universidade Estadual de Londrina).

Bontempo, E. (1986). Psicologia do brinquedo: Aspectos teóricos e metodológicos. São Paulo: NovaStella.

Domingos, N. A. M. (1993). Preparo para cirurgia: Teste de programas psicológicos na redução de ansiedade de crianças e mães. Dissertação de Mestrado(PUC). Campinas.

Gaetan, R. H. M. C. (1997). O estresse na criança. Seminário de Psicologia da Saúde. São José do Rio Preto: UNORP.

Gesell, A. (1985). A criança dos 0 aos 5 anos. São Paulo: Martins Fontes. 
Guimarães, S. S. (1988, Mai-Ago.). A hospitalização nainfância. Psicologia: Teoria e pesquisa, 4 (2), 102-112, Universidade de Brasília.

Laurent, A. P., \& Rumeu, O. L. (1990). Programas para la preparación a la hospitalización infantil. In: J.M. Buceta \& A. M. Bueno, Modificación de conductay salud: Perspectivas actuales en la aplicación de tratamientos de psicologia [s.1]. Eudema.

Lindquist, I. (1975). Ludoterapia no hospital. Artigo extraído de Congresso Pediátrico, São Paulo.

Lindquist, I. (1992). Brincar no hospital. In: A. Friedmann, $O$ direito de brincar: A brinquedoteca (pp.123-133). São Paulo: Scrita, Abrinq.

Lindquist, I. (1993). A criança no hospital: Terapia pelo brinquedo. São Paulo: Scritta.

Méndez, F. x., Ortigosa, J. M., \& Pedroche, S. (1996). Preparación a la hospitalización infantil (I): Afrontamiento del estrés. Psicologia conductual, 4, (2), 193-209, Murcia.
Ortigosa, J. M., Méndez, F. x., \& Quiles, M. J. (1996). Preparación a la hospitalización infantil (II):modelado filmado. Psicologia conductual, 4, (2), 211-230, Murcia.

Piaget, J. (1964). Seis estudos de psicologia. Rio de Janeiro:Forense-Universitária.

Ribeiro, C. A. (1998, Abr.). O brinquedo terapêutico na assistência à criança hospitalizada: Significado da experiência para o aluno de graduação em enfermagem. RevistadaEscolade Enfermagem, 32, (1), 73-79, USP.

Saggese, E. S. R., \& Maciel, M. de A. (1997). Projeto saúde e brincar. Rio de Janeiro: Instituto Femandez Figueira daFundação Oswaldo Cruz.

Santos, S.M. P. (Org.) (1997) Olúdico na formação do educador. Petrópolis: Vozes.

Whaley, L. F., \& Wong, D. L. (1989). Enfermagem pediátrica: Elementos essenciais à intervenção efetiva (2" ed.). Rio de Janeiro: Guanabara Koogan. 